\title{
Optimizing aerodynamic lenses for single-particle imaging
}

\author{
Nils Roth ${ }^{\mathrm{a}, \mathrm{b}}$, Salah Awel ${ }^{\mathrm{a}, \mathrm{c}}$, Daniel A. Horke ${ }^{\mathrm{a}, \mathrm{c}}$, Jochen Küpper ${ }^{\mathrm{a}, \mathrm{b}, \mathrm{c}}$ \\ ${ }^{a}$ Center for Free-Electron Laser Science, Deutsches Elektronen-Synchrotron DESY, Notkestrasse 85, 22607 Hamburg, Germany \\ ${ }^{b}$ Department of Physics, Universität Hamburg, Luruper Chaussee 149, 22761 Hamburg, Germany \\ ${ }^{c}$ The Hamburg Center for Ultrafast Imaging, Universität Hamburg, Luruper Chaussee 149, 22761 Hamburg, Germany
}

\begin{abstract}
A numerical simulation infrastructure capable of calculating the flow of gas and the trajectories of particles through an aerodynamic lens injector is presented. The simulations increase the fundamental understanding and predict optimized injection geometries and parameters. Our simulation results were compared to previous reports and also validated against experimental data for $500 \mathrm{~nm}$ polystyrene spheres from an aerosol-beamcharacterization setup. The simulations yielded a detailed understanding of the radial phase-space distribution and highlighted weaknesses of current aerosol injectors for single-particle diffractive imaging. With the aid of these simulations we developed new experimental implementations to overcome current limitations.
\end{abstract}

Keywords: aerodynamic lens, aerosol, coherent diffractive imaging, simulation, single-particle imaging

\section{Introduction}

Single-particle diffractive imaging (SPI) is one of the key applications enabled by the advent of x-ray free-electron lasers (XFELs) [1, 2]. Short-duration XFEL pulses were predicted to allow the collection of diffraction patterns from radiation-sensitive samples without resolution limitations due to radiation damage [3, 4, although some open questions remain [4] 6]. A series of two-dimensional diffraction patterns of randomly oriented isolated particles can be used to reconstruct the full three-dimensional structure, without the need for large highly ordered crystalline samples [2, 7, 8].

As every intercepted particle is destroyed by the intense x-ray pulse [9], a new and preferably identical sample particle has to be delivered into every pulse. This can be achieved with aerosolized particle beams, which, furthermore, offer significantly reduced background levels compared to liquid jet based delivery methods [10, 11]. The most widespread aerosol injectors for SPI experiments are aerodynamic lens stacks (ALS) [1, 12]. However, other aerosol injectors, e.g., convergent nozzles, have also been demonstrated [11, 13, 14. One of the limiting factors for SPI is the collection of a sufficient number of strong

\footnotetext{
* Author to whom correspondence shall be addressed:

Email address: jochen.kuepper@cfel.de (Jochen Küpper)

$U R L$ : https ://www. controlled-molecule-imaging.org (Jochen Küpper)
} 


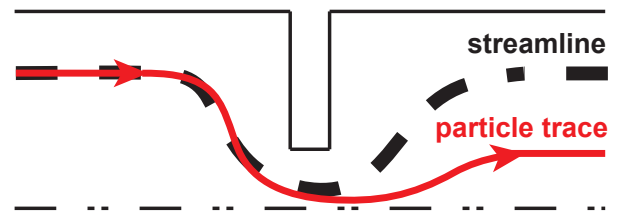

Figure 1: Schematic of particles being contracted toward the centerline by a gas stream through an orifice. The orifice is cylindrical symmetric around the dashed line. The trajectories of particles past the orifice vary from the streamlines due to particle inertia, leading to an aerodynamic lensing effect.

diffraction patterns [15, 16]. Overcoming this limitation requires the delivery of high-density particle streams in order to maximize the number of x-ray pulses intersecting a particle and producing a measurable diffraction pattern. Using current aerosol injectors, hit fractions, i. e., the fraction of x-ray pulses that hit at least one particle, up to $79 \%$ could be achieved [12. However, this contains pulses interacting with multiple particles, faint hits far from the x-ray maximum intensity, as well as hits from background particles. This leads to hit rates, i. e., usable diffraction patterns containing a bright image from a single isolated target particle, of below $5 \%$ and, hence, long measurement times and excessive sample consumption. Furthermore, these current studies have been undertaken with x-ray-focal-spot sizes on the order of a few micrometers. This comparatively soft focusing of the XFEL pulse does not yield the photon intensity required for measurable SPI diffraction signal to high scattering angles or from small samples [17]. This requires nanofocused x-ray beams with focal spot sizes on the order of $100 \mathrm{~nm}$, where the hit-rate achievable with current aerosol injectors is typically below $0.05 \%$.

As particles distribute stochastically in the aerosol beam, the probability for them to be within the x-ray interaction volume depends on the local particle density, necessitating highly collimated or focused particle streams. Robinson predicted in 1956 that in real, irrotational and incompressible gas flow past an obstacle, the density of small particles within the flow can increase while passing the obstacle [18. This mathematical description was later extended and used, supported by numerical simulations, to describe particles flowing in a tube through an orifice [19. Under the right conditions, in what is now known as an aerodynamic lens $(\mathrm{ADL})$, the particles concentrate at the center of the tube, as illustrated in Figure 1 .

A detailed numerical characterization of an individual ADL was presented in 2002 [20], which was later extended to an entire ALS [21. Numerical simulations for an ALS to focus particles with diameters below $30 \mathrm{~nm}$ [22] led to a simple design tool that predicts the required lens dimensions to focus a specified range of particle sizes at given flow conditions [23. Based on this, further numerical simulations have adapted ALS to specific needs [24, 25]. Although ALS have been used for, e.g., ultrafast electron imaging experiments on nanoparticles [26], they are predominantly used in aerosol mass spectrometry [27]. Here, the main goal is to contract a large range of particle sizes and ensure a high transmission. The "Uppsala" ALS, a widely used standard injector for SPI [12, was designed based on the same principle. Therefore, it can deliver collimated 
particle beams for a large range of particle sizes $(0.1-3 \mu \mathrm{m})$ without changing the apparatus. However, the possibility to optimize for a specific particle size is limited, and the pressure before the ALS is the only tunable parameter.

These design principles differ from the requirements of SPI experiments, where highly collimated beams of only one particular particle size are needed, and even desirable in order to increase sample purity. Additionally, the final particle beam diameter should be matched to the x-ray focal size. To enable the transmission of a wide range of particle sizes requires the use of several orifices within the ALS. This increases the complexity of the setup and the individual orifices are designed for different particle sizes, making some of them counterproductive for producing a high-density beam of a well-defined particle size.

Here, we present a detailed numerical simulation environment to understand and to quantitatively model the underlying fundamental processes occurring within an ALS and to further optimize these systems to meet the requirements of SPI experiments. In particular, we aim to design an ALS optimized for focusing a single particle size to the smallest possible beam diameter, while keeping the experimental setup simple and easily adaptable for different samples.

\section{Methods}

\subsection{Numerical Simulation}

Optimizing the geometry of an ADL requires investigating a large parameter space, such as dimensions of orifices and transport tubes, making experimental characterization and optimization impractical. Instead, we implemented numerical simulations to predict the behavior of particles within the ADL. Furthermore, these simulations allow the extraction of phase-space distributions of particles at any position within the device. Within all simulations we assumed that, (i) particles in the flow field have no influence on the flow field itself and (ii) particles do not interact with each other. This implies that the flow field and particle trajectories can be calculated separately, and that each particle can be simulated independently. These assumptions significantly reduce computational cost, and are easily justified considering the typical pressures in an ALS. The helium (or carrier gas) pressure is $\sim 1 \mathrm{mbar}$ (number density of $\sim 10^{16}$ atoms $/ \mathrm{cm}^{3}$ ), while the density of particles usually does not exceed $10^{10}$ particles $/ \mathrm{cm}^{3}$.

For an accurate description of the ALS and produced particle beams, the phase-space distribution of particles at the inlet of the injector is a crucial parameter. This distribution is typically defined by either the aerosol source or, more commonly, by a differential pumping stage used to reduce the gas-load and to control the pressure upstream of the ALS. A common arrangement for such a pumping stage is a set of two skimmers, oriented with the tips facing each other, as described in detail in subsection 2.2 and shown schematically in Figure 2. Since there is no experimental data available for the phase-space distribution of 
particles before they enter the ALS, the initial particle conditions are evaluated through simulations of the flow through the skimmers.

\subsubsection{Flow field}

We simulated the flow field of the carrier gas using a finite-element solver [28] to solve the Navier-Stokes equations. The geometry and flow were assumed to be axisymmetric about the central axis, and the flow solved in two dimensions $(r, z)$. The flow was treated compressible and viscous, and the calculation iterated until converged to a steady-state solution. Additional properties of the flow field are indicated by three dimensionless quantities, the Reynolds number Re, the Knudsen number $K n$, and the Mach number $M a$. $R e$ is defined as the ratio of inertial to viscous forces. Typically, Reynolds numbers in an ALS are below 10, indicating that no instabilities are present in the flow field, which we thus solve assuming laminar flow. $K n$ is defined as the ratio of the mean free path to a characteristic length. Inside the ALS the pressure is usually on the order of $1 \mathrm{mbar}$, corresponding to a mean free path around $70 \mu \mathrm{m}$ for helium at room temperature. Compared to the dimensions of apertures (a few $\mathrm{mm}$ ) this results in $K n<0.01$ and there are enough collisions with the background gas to treat the flow as continuum and the Navier-Stokes equations hold. Upstream of the ALS, before and throughout the two skimmer setup, the pressure is even higher, hence $K n \ll 0.01$. In the vacuum chamber, on the other hand, at helium pressures of $10^{-2}$ mbar or less, the mean free path is around $10 \mathrm{~mm}$, hence $K n>0.01$, and the continuum flow model breaks down. With such a large mean free path particles rarely collide with the background gas, such that there is no momentum exchange with the flow field anymore. In between these regimes there is a transition region which is difficult to model. However, this transition between regimes occurs rapidly after particles exit the tip of the injector, and here we assumed a sudden stop of the continuum flow regime and an immediate change to the molecular flow regime. In the latter, particles were assumed to propagate collision-less and with constant velocity. The ratio of the velocity of the flow to the speed of sound in the fluid is the Mach number, $M a$. For $M a>0.3$ effects due to the compressibility of the fluid start to occur, such as pressure waves and cooling of the fluid. Here, such high $M a$ are reached between the two skimmers and downstream of the injector tip. Pressure waves are still properly described by the Navier-Stokes equations. However, our numerical simulation approach, finite element methods, necessitates the use of stabilization methods, which add artificial diffusion in order to avoid numerical instabilities, e.g., oscillations in the solution. While these might wash out the position and velocity of the potentially occurring pressure waves, the position of the second skimmer is closer to the first skimmer exit than the calculated distance of the Mach disc, where these pressure waves are supposed to be located. In Addition, downstream of the injector tip and between the two skimmers particles are fast and have a high intertia. Hence, the effect of the spiky features caused by high $M a$ are assumed to have a limited influence on the overall particle trajectories, especially at the injector tip, where the continuum flow breaks. Nevertheless an accurate treatment of the flow including thermodynamic coupling might be able to further 
improve the simulation results.

\subsubsection{Particle traces}

Particle trajectories were simulated, within a given steady-state flow field, with a homebuilt python code that uses a real-valued variable-coefficient ordinary-differential-equation solver. The code interpolates the given pressure and velocity fields and calculates the forces, described by Stokes' law, acting on a particle of given size at each time step. Additional corrections can arise depending on the particle's Reynolds $\left(R e_{\mathrm{p}}\right)$ and Knudsen $\left(K n_{\mathrm{p}}\right)$ numbers. These are defined identical to the fluid case, but with the characteristic length given by the particle diameter. $R e_{\mathrm{p}}$ is very small inside the lens $\left(R e_{\mathrm{p}}<1\right)$ and can be neglected. $K n_{\mathrm{p}}$, however, cannot be ignored as at low gas densities the mean free path is larger than the particle diameter, leading to a decreased drag force due to the reduced number of collisions. This is taken into account by the Cunningham slip-correction factor $C_{\mathrm{c}}$ [29], which gives the drag force as

$$
\begin{aligned}
F_{\mathrm{drag}} & =\frac{3 \pi \mu d_{\mathrm{p}}(\vec{U}-\vec{u})}{C_{\mathrm{c}}} \\
\text { with } C_{\mathrm{c}} & =1+K n_{\mathrm{p}}\left(c_{1}+c_{2} \cdot e^{c_{3} / K n_{\mathrm{p}}}\right)
\end{aligned}
$$

Here, $\vec{U}$ is the local velocity of the flow field, $\vec{u}$ the particle velocity and $d_{\mathrm{p}}$ the particle diameter. The empirical coefficients $c_{1}=1.2310, c_{2}=0.4695$ and $c_{3}=-1.1783$ are taken from the literature [29]. This model describes the interaction of a particle with a continuum flow field. In reality, however, the particles interact via single collisions with the carrier gas. This leads to diffusion and an additional random walk of the particles around their trajectory. This is numerically described by a Brownian-motion force $F_{\mathrm{b}}$, which is added to the drag force [30]

$$
F_{\mathrm{b}}=m_{\mathrm{p}} \vec{G} \sqrt{\frac{\pi S_{0}}{\Delta t}} \quad \text { with } \quad S_{0}=\frac{216 \mu k T}{\pi^{2} d_{\mathrm{p}}^{5} \rho_{\mathrm{p}}^{2} C_{\mathrm{c}}},
$$

where $\vec{G}$ is a vector of zero mean, unit variance, independent Gaussian random numbers, $\Delta t$ the time step size of the solver, $k$ the Boltzmann constant, $T$ the temperature of the carrier gas, $m_{\mathrm{p}}$ the particle mass, $d_{\mathrm{p}}$ the particle diameter and $\rho_{\mathrm{p}}$ the particle density. Since the flow field was treated axisymmetrically, the Brownian force was restricted to have axial and radial components. Particle trajectories are calculated until they reach the boundary of the flow field. This happens either when they are successfully transmitted to the end of the flow field downstream of the geometry or when they touch the wall of the geometry and are considered lost due to impaction.

\subsection{Experimental Setup}

To validate our simulations and the ability to predict ALS behavior, we benchmarked them against experimental data. The experimental setup and data analysis has been described in detail previously [14]. 


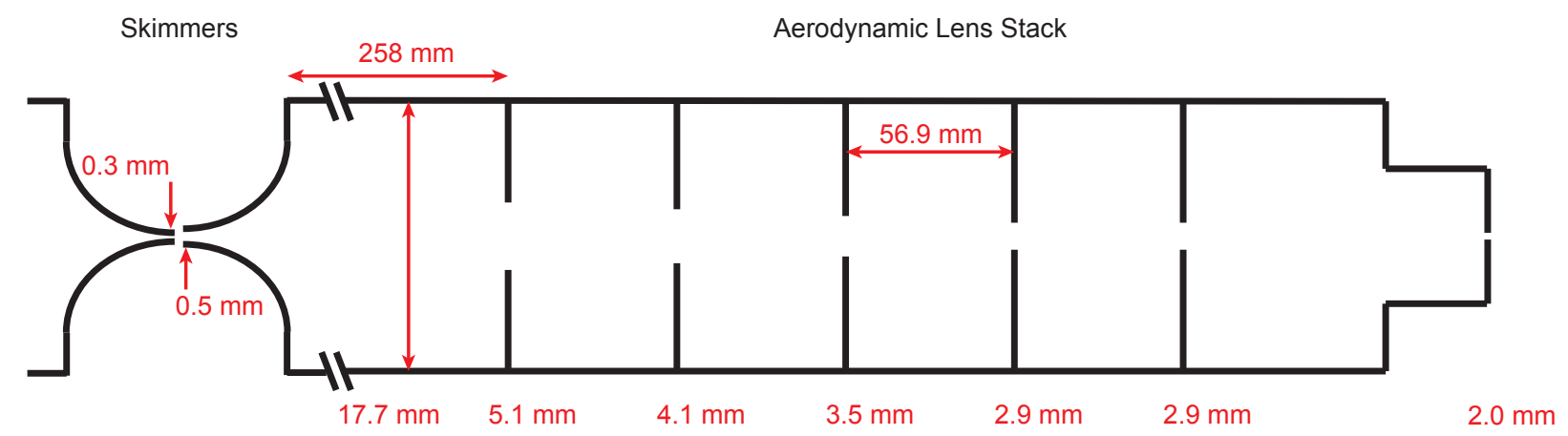

Figure 2: Sketch (not to scale) of the double skimmer setup and the ALS injector with its 6 orifices. The dimensions given below the orifices refer to the inner diameter.

Briefly, we used a gas dynamic virtual nozzle to aerosolize an aqueous solution of $500 \mathrm{~nm}$ sized polystyrene spheres (Thermo Fisher Scientific) into a nebulization chamber [10, 31]. Particles then passed a set of two skimmers, with inner diameters of $0.3 \mathrm{~mm}$ and $0.5 \mathrm{~mm}$, respectively, placed $2 \mathrm{~mm}$ apart, as sketched in Figure 2, Evacuating the volume between the skimmers allows control over the pressure before the ALS while minimizing particle losses. After the ALS particles were illuminated with a Nd:YLF laser (Spectra Physics Empower ICSHG-30, 527 nm, pulse duration $100 \mathrm{~ns}$, pulse energy $20 \mathrm{~mJ}$ ) and scattered light collected on a translatable high-frame-rate CMOS camera (Photron SA4) using a $5 \times$ infinity-corrected objective (Mitutoyo, numerical aperture 0.14). The geometric dimensions of the ALS used are specified in Figure 2.

\subsection{Theoretical description of the experimental setup}

For the theoretical model, we first calculated flow fields within which particles are then propagated. When trying to simulate the entire apparatus, containing differential-pumping skimmers and the ALS, we encountered convergence problems and no steady solution was found. Therefore, we retreated to evaluate the flow fields for the ALS and the skimmer setup separately, but made sure that they are consistent.

The effect of the skimmers was approximated by simulating the flow field through the upper skimmer, simulating particle trajectories and retaining only particles with a radial position smaller than $250 \mu \mathrm{m}$ at a position $2 \mathrm{~mm}$ downstream of the skimmer tip, representing those that would enter into the second skimmer. The purpose of the upper skimmer is to accelerate particles, such that their momentum is high enough to enter the lower skimmer without being significantly disturbed by the flow field between the skimmers, where excess gasload is evacuated. Boundary conditions constrained the inlet mass flow through the skimmer to $30 \mathrm{mg} / \mathrm{min}$, comparable to experimental conditions. The outlet was defined as a semi-circle at the tip of the skimmer with a $2 \mathrm{~mm}$ radius, corresponding to the distance between the skimmers. Along this semicircle the pressure was constrained to experimentally measured values. Particles were assumed to be spheres with $500 \mathrm{~nm}$ diameter and a density of $1050 \mathrm{~kg} / \mathrm{m}^{3}$ (polystyrene), with an initial uniform distribution at the 
entrance plane of the skimmer. The longitudinal and radial velocities of the particles are set to the flow velocity at their initial position. The recorded final phase-space distributions of transmitted particles are used to define the initial particle phase-space distribution at the ALS.

To simulate the ALS we introduced boundary conditions for the pressure at the inlet and outlet. The former was defined as the entrance plane at the beginning of the ALS tube, and pressures set to experimental values. The outlet was defined as a semi-circle at the tip of the ALS into vacuum with radius $1 \mathrm{~mm}$, corresponding to the radius of the final aperture of the ALS. The pressure along this semicircle was assumed to be $10^{-2}$ mbar. Reducing this pressures further does not change the dynamics in the flow, since they depend on pressure difference, which is already dominated by the two-orders-of-magnitude higher pressure inside the ALS. With these boundary conditions we calculated a steady flow field for every inlet pressure. The initial phase-space distributions of particles were taken from the skimmer simulations, but with the initial longitudinal position of all particles set to the entrance plane at the beginning of the ALS tube.

We simulated $10^{5}$ particles per upstream pressure. Final particle trajectories contain the axisymmetric two dimensional position of particles throughout and after the ALS. In the experiment, we probed the particle beam orthogonal to the propagation direction by projecting it onto the imaging plane of a camera [14]. Hence, the simulated radial particle beam distribution was projected in silico onto a two dimensional imaging plane for comparison.

\section{Results \& Discussion}

\subsection{Validation against literature simulations}

We first validated our simulation environment against the simulations by Wang et al. 23]. We replicated the geometry and conditions of the original publication and simulated particle trajectories. Figure 3 shows the local gas velocity and pressure as well as the velocity of 1, 10 and $40 \mathrm{~nm}$ particles along the centerline of the ALS. Our results show excellent agreement with the previously published simulations, c. f. Figure $2 \mathrm{~b}$ of reference 22, and only deviate slightly in the region outside the actual ALS $(z>7 \mathrm{~cm})$. These deviations can be explained by the different outlet boundary conditions.

\subsection{Particle Beam Characterization}

\subsubsection{Experimental Results}

All measurements were conducted with the setup described in subsection 2.2 , and the only parameter varied was the pressure upstream of the ALS. The particle stream was imaged $8 \mathrm{~mm}$ downstream of the injector tip and data collected for $\sim 10 \mathrm{~min}$ at each pressure, corresponding to $\sim 10^{5}$ imaged particles. For comparison with theoretical results, we determined the particle beam width containing $90 \%$ (70\%) of all particles, denoted $D_{90}\left(D_{70}\right)$. Measured beam widths are shown in Figure 4 a for upstream pressures in the range 0.66 to $2.0 \mathrm{mbar}$. 
The full distributions for three characteristic pressures are shown in Figure $4 \mathrm{~b}-\mathrm{d}$ (black lines). It is evident from the experimental data that the particle beam width decreases with increasing upstream pressure until a critical value, here $\sim 1.2$ mbar, after which no dependence on pressure is observed anymore and the produced beam width remains practically constant.

\subsubsection{Theoretical Results}

In order to simulate the resulting particle beam downstream of the ALS, it is necessary to compute the initial phase-space distribution of particles entering the ALS by simulating the first skimmer as detailed above. An example of a velocity field inside and such a radial phase-space distribution of particles after the first skimmer is shown in Figure 5 a and b, respectively. The radial position is cut at $250 \mu \mathrm{m}$, as only these particles enter into the second skimmer. A large fraction of particles is contained within a small region of phase space at radial velocities between 10 and $15 \mathrm{~m} / \mathrm{s}$.

Simulated radial phase-space density distributions of particles through the flow-field shown in Figure 6

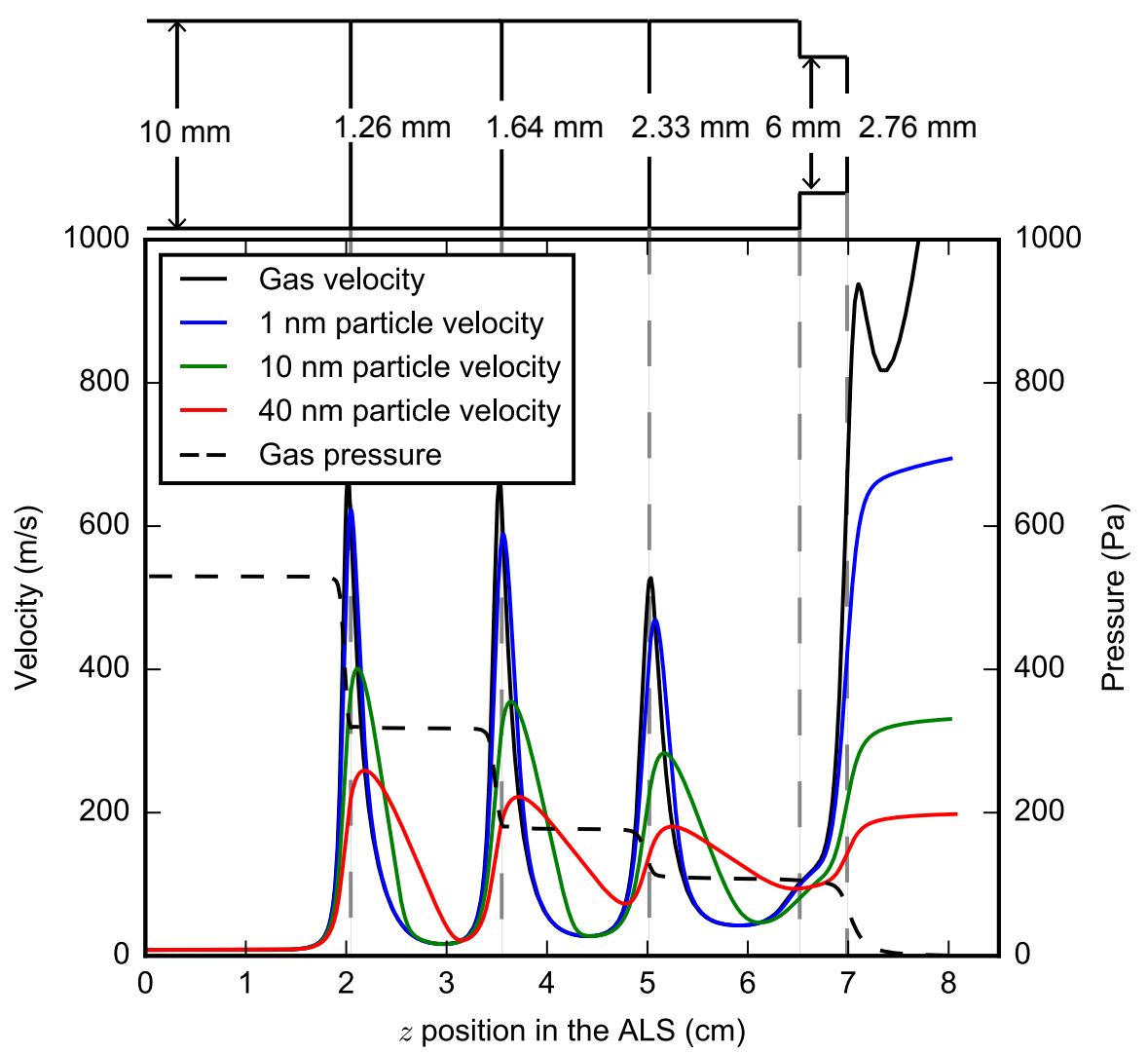

Figure 3: Simulation of gas pressure, gas velocity and particle velocity of 1,10 and 40 nm particles along the centerline of the ALS investigated in 22, and shown at the top of the figure. Our simulation accurately reproduced the results from the original publication [22, Figure 2]. 

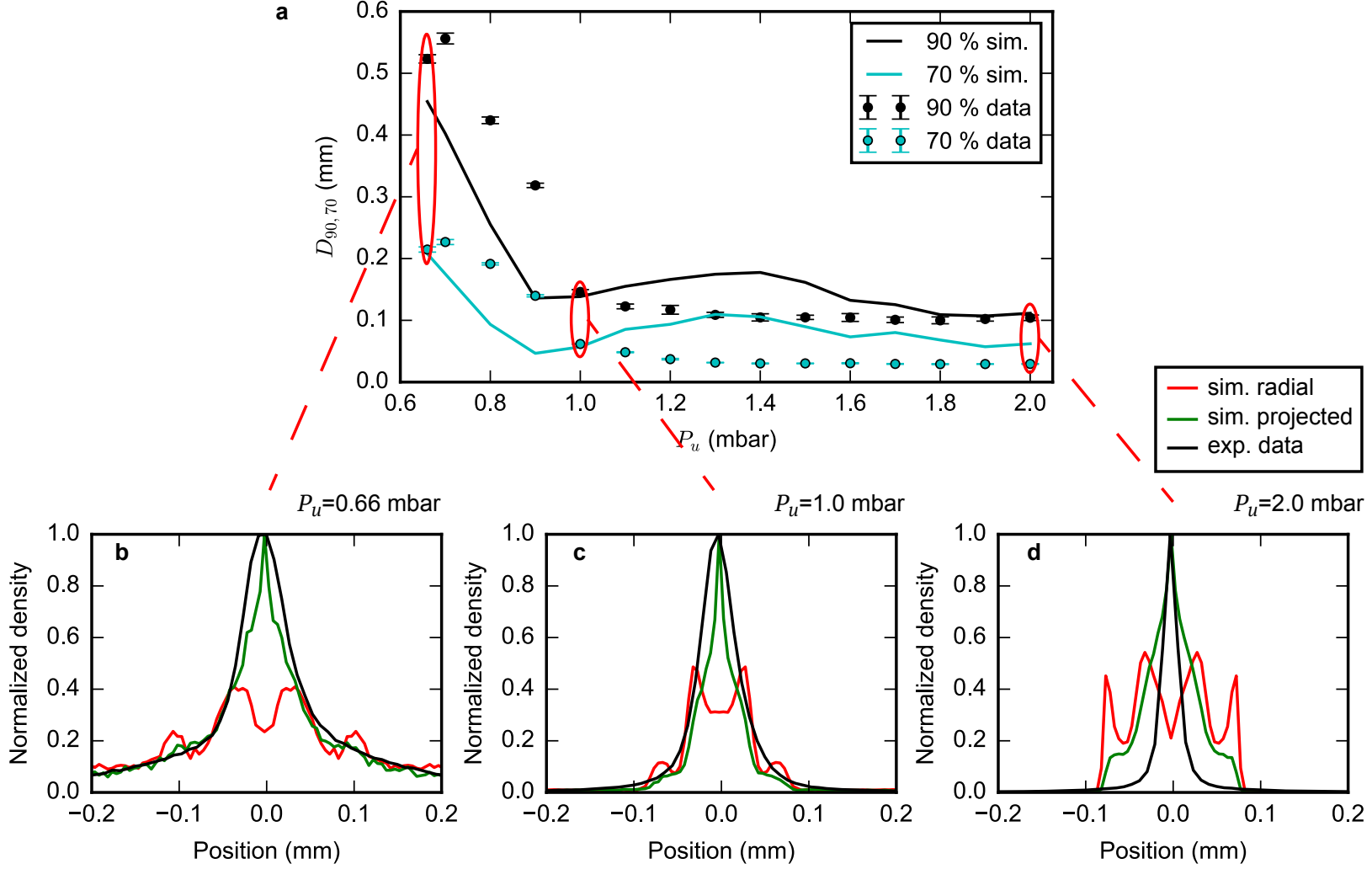

Figure 4: Comparison of experimental, i. e., projected, and simulated, i. e., projected in silico, beam width for 500 nm particles as a function of inlet pressure $P_{u}$. The errorbars represent the statistical standard error (a). Comparison of experimental, i. e., projected (black line), simulated radial (red line), and simulated projected (green line),

particle profiles for three distinct upstream pressures of the ALS, 0.66 mbar (b), 1.0 mbar (c) and 2.0 mbar (d).

at various positions within the ALS are displayed in Figure 7 for two different upstream pressures. The left hand side of Figure 7 corresponds to 0.66 mbar and the right hand side to 2.0 mbar. The top images show the initial distribution (beginning of the injector), while all others contain only successfully transmitted particles, which are shown at three positions; at the beginning of the ALS (c and d), just before the first ADL (e and $\mathrm{f}$ ), $1 \mathrm{~mm}$ after exiting the ALS ( $\mathrm{g}$ and $\mathrm{h}$ ) and at the particle focus $10 \mathrm{~mm}$ and $5 \mathrm{~mm}$ after exiting the ALS, respectively (i and j). Whereas the initial phase-space distributions of particles are nearly identical for the two pressures, the number of particles transmitted and their phase-space distributions throughout and after the ALS are markedly different. While for high $P_{\mathrm{u}}$ all particles are transmitted, this is not the case at low $P_{\mathrm{u}}$. Here, the initial phase-space distribution of successful transmitted particles exhibits a cutoff at around $6 \mathrm{~m} / \mathrm{s}$ absolute radial velocity (Figure $7 \mathrm{c}$. We rationalize this with the pressure scaling of the drag force. Particles at lower pressures, and hence lower drag force, are not slowed down sufficiently in the 

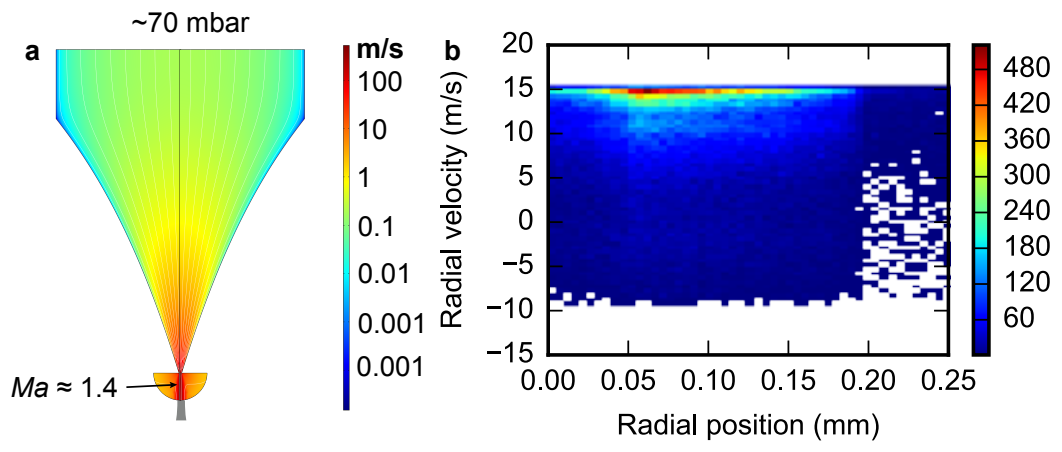

Figure 5: Simulated flow field with streamlines inside the upstream skimmer. The logarithmic color scale corresponds to the flow speed. The grey area indicates the position of the lower skimmer. (a). Histogram of the radial position and velocity of particles $2 \mathrm{~mm}$ downstream from the skimmer tip (b).

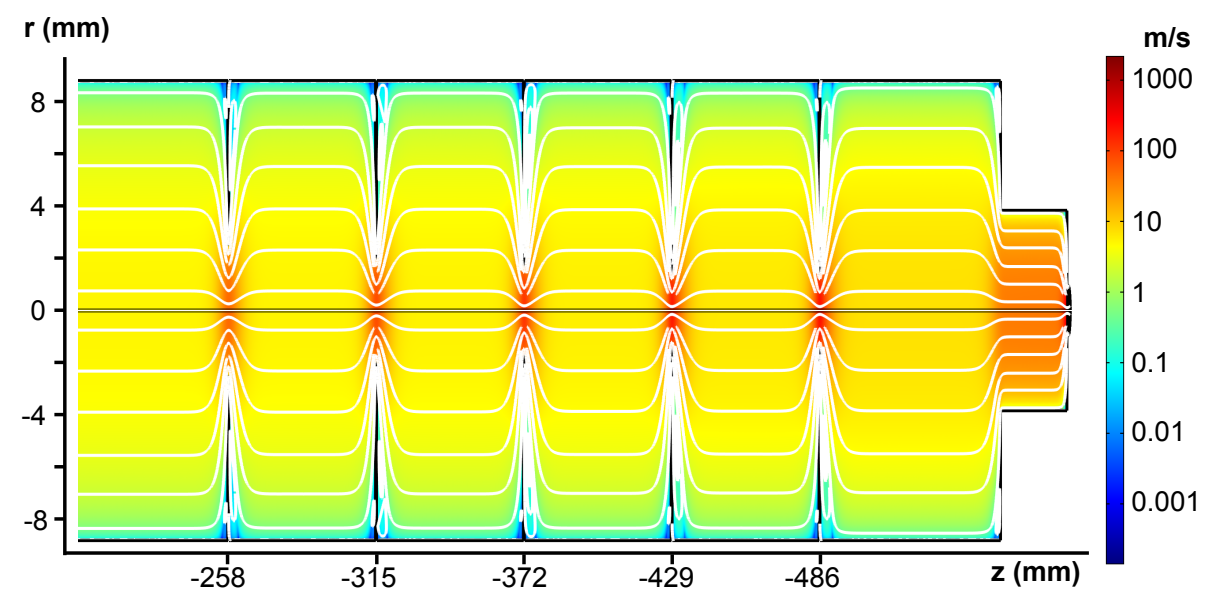

Figure 6: Simulated flow field with streamlines inside the ALS injector. The $r$-axis has been scaled by a factor of 10 . The logarithmic color scale corresponds to the flow speed. 


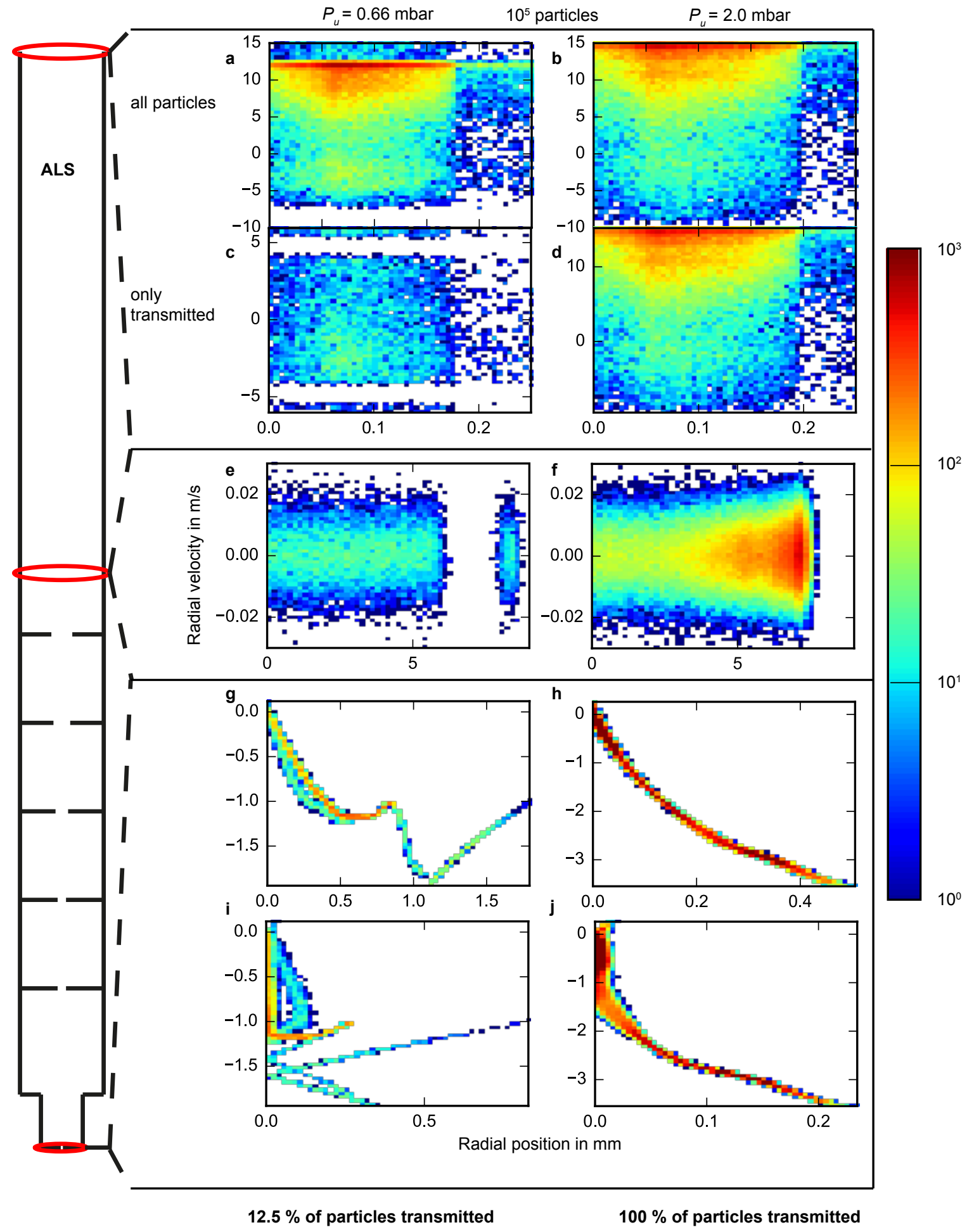

Figure 7: Histograms of the radial phase-space distribution for $500 \mathrm{~nm}$ particles at various positions in the ALS for $P_{u}=0.66 \mathrm{mbar}$ $(\mathbf{a}, \mathbf{c}, \mathbf{e}, \mathbf{g}, \mathbf{i})$ and $P_{u}=2.0$ mbar $(\mathbf{b}, \mathbf{d}, \mathbf{f}, \mathbf{h}, \mathbf{j})$. a, b show the distributions for all particles at the inlet of the ALS. All other distributions show only particles successfully transmitted through the ALS and are taken at a position at the inlet (c, d), before the first lens $(\mathbf{e}, \mathbf{f})$, after the ALS $(\mathbf{g}, \mathbf{h})$ and at the distance of highest density, $10 \mathrm{~mm}(\mathbf{i})$ and $5 \mathrm{~mm}(\mathbf{j})$ downstream of the outlet, as indicated by the red lines. 
radial direction, such that they collide with the wall of the ALS tube and are lost. This is not the case at $P_{\mathrm{u}}=2$ mbar, where all particles are slowed down before they reach the wall and are transmitted. The radial velocity of the particles before the first lens is now essentially zero and most particles lie within $\pm 0.02 \mathrm{~m} / \mathrm{s}$. The phase-space densities in Figure $7 \mathrm{e}$ and $\mathrm{f}$ show that particle radial positions are spread over the entire ALS tube, and that at higher pressures (f) the majority of particles are at large radii. These particles at large radial positions correspond to those with an initially large radial velocity. In the low pressure case (e) high-radial-velocity particles collided with the wall and were lost. This correlation between initial radial velocity and radial position is, furthermore, evident for the low pressure case (Figure $7 \mathrm{c}$ and e), where an initially empty area of velocity space (between 4 and $5 \mathrm{~m} / \mathrm{s}$ ) appears as an empty area in position space (around $7 \mathrm{~mm}$ ) before the first ADL. Thus the acceptance of the ALS depends on the upstream pressure, i. e., the flow field, and in the low pressure case the transmission and behavior of the ALS depend critically on the radial particle position before the first ADL, hence the initial radial particle velocity. This position-dependent behavior of an ADL will be further investigated in subsection 3.3

In both pressure regimes the distribution of final radial positions (Figure $7 \mathrm{~g}$ and $\mathrm{h}$ ) is concentrated toward the centerline in comparison to the distribution before the first lens (Figure 7 e and $\mathrm{f}$ ) and the final radial velocity distribution is narrower, hence, the particle beams are more collimated than the inlet distributions (Figure $7 \mathrm{a}$ and b). However, in the high pressure case particles are confined to significantly smaller radii corresponding to $D_{70}=226 \mu \mathrm{m}$ at this position in comparison to $D_{70}=815 \mu \mathrm{m}$ for the low pressure case. The particles radial velocity is predominantly negative after the lens, corresponding to a motion toward the centerline, i. e., the particle beam converges. Particles at higher radial positions have a greater negative radial velocity and, therefore, a higher density of particles will be achieved downstream of the injector. In the 0.66 mbar case the highest density is achieved $10 \mathrm{~mm}$ after the injector outlet, while for $2 \mathrm{mbar}$ it is $5 \mathrm{~mm}$ downstream of the outlet. The corresponding phase-space distributions are shown in Figure $7 \mathrm{i}$ and $\mathrm{j}$. Note that from $1 \mathrm{~mm}$ downstream of the injector (the end of the calculated steady-state flow field) onwards the particles are propagated straight without any forces acting on them.

The final phase-space distribution is predominantly defined by the last aperture, as the radial velocity upstream of each ADL is centered around 0, see Figure $7 \mathrm{e}$ and $\mathrm{f}$. In order to qualitatively rationalize the observed distributions we consider the radial-position dependence of the radial velocity and total speed of the gas flow before (Figure $8 \mathrm{a}$ and after Figure $8 \mathrm{~b}$ the last lens in the ADL. The radial velocity of the flow changes throughout the orifice, from a contraction towards the centerline, i. e., negative radial velocities in Figure $8 \mathrm{a}$, to an expansion afterwards, positive radial velocity in Figure $8 \mathrm{~b}$. This dramatic change is caused by the significantly different pressure regimes, inside the ADL versus outside the ADL. Since the drag force is proportional to the difference in particle velocity and flow field velocity, see (1), the force acting on a particle in the radial direction is proportional to the radial flow velocity. In Figure $8 \mathrm{c}$ we show the particle phase-space distribution before the last orifice, i. e., at the same position as the gas flow distribution shown 

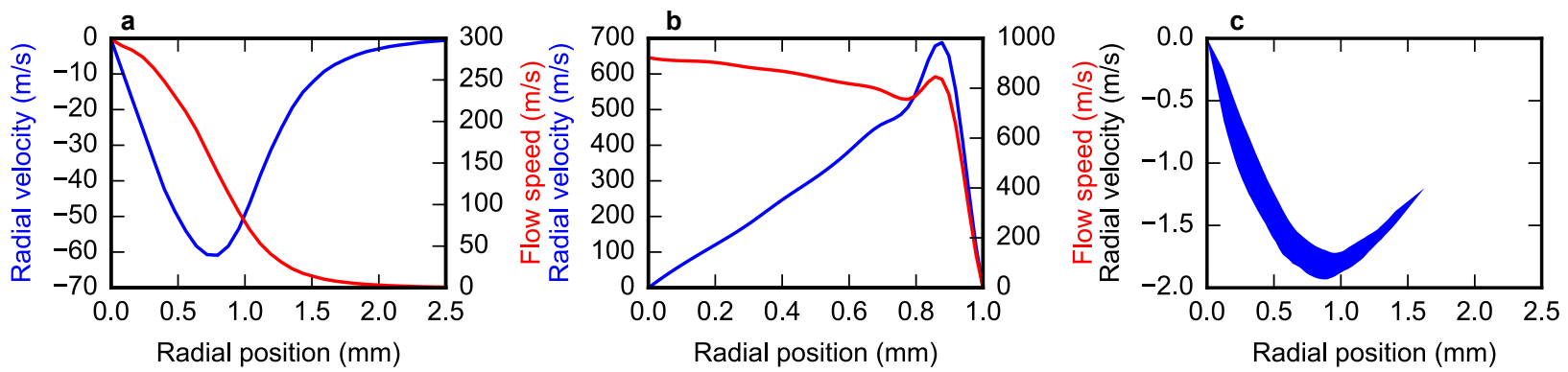

Figure 8: Radial velocity and speed of the helium flow as a function of radial position for 0.66 mbar upstream pressure at a position $0.2 \mathrm{~mm}$ before (a) and $0.5 \mathrm{~mm}$ after (b) the last orifice in the ALS injector. Phase-space distribution of $500 \mathrm{~nm}$ particles $0.2 \mathrm{~mm}$ before the last orifice in the ALS injector at a 0.66 mbar upstream pressure (c).

in Figure $8 \mathrm{a}$, A clear correlation is observed between the radial gas-flow velocity, blue line in Figure 8 a, and the particle phase-space distribution. As the radial velocity of the gas is changing rapidly on passing the orifice, one might expect a similar effect on the radial velocity distribution of particles. However, since particles carry a significant amount of inertia, they cannot follow this rapid change in gas-flow and the particle phase-space distribution even after the last aperture (as shown in Figure $7 \mathrm{~g}$ ) is still dominated by the distribution before the orifice. One noticeable difference, however, is an increase of radial velocity around the position $0.8 \mathrm{~mm}$. We attribute this to the rapid expansion of gas after the last aperture, which peaks at this radial position (Figure $8 \mathrm{~b}$ and, hence, accelerates particles most at this distinct radius, leading to the observed local maximum in the particle radial velocity around $0.8 \mathrm{~mm}$.

Where within the general shape of the phase-space distribution particles are located (i. e., the intensity information missing in Figure 8 c is dependent on the radial position of particles upstream of an ADL. Since, in the high pressure case, no particles are at radial positions above $\sim 0.5 \mathrm{~mm}$, only the initial falling edge at small radial positions is represented in the phase-space distribution in Figure $7 \mathrm{~h}$

\subsubsection{Comparison of Simulation and Experiment}

We compared the simulated results with experimental data by reproducing the pressure dependence of the particle-beam width evaluated $8 \mathrm{~mm}$ downstream of the ALS tip, as shown in Figure 4 a. The simulations clearly reproduced the experimental observation, with a sharp drop in beam diameter as the pressure is increased, until a plateau is reached at $\sim 0.9$ mbar. Full radial distributions of particles are shown in Figure 4 b-d for three inlet pressures and exhibit an overall good agreement with the experimental data. Some deviations are, however, observed. A slight pressure offset for the location of the sharp drop is most likely due to the $\pm 15 \%$ uncertainty of the pressure gauges used (Pfeiffer Vacuum, TPR 280). Moreover, the simulations overestimate the particle beam size in the plateau region, which could be due to the limited illumination area of the laser used for particle detection [14. If particles far from the center were not correctly identified, 
this would lead to lower than expected experimental values for $D_{70}$ and $D_{90}$. On the other hand, for very high particle densities, there is a probability that the image analysis software cannot distinguish individual particles anymore. This would lead to a decreased particle density detected in the central region.

We also note that our simulation might oversimplify the occurring physical processes, e.g., particles are assumed to have no collisions $1 \mathrm{~mm}$ downstream of the last aperture, whereas there are some experimental indications that particles still accelerate in this region [32. In addition, the used Cunningham correction factors, see subsubsection 2.1.2, were derived for air instead of helium. While this might render it difficult to computationally reach high accuracy, the overall good agreement justifies the use of these models, and our simulation infrastructure in general, to understand and predict ALS behavior.

\subsection{Radial distribution analysis}

In SPI experiments the interaction volume is a cylinder, representing the x-ray beam volume, through the three dimensional particle beam. Therefore, the vast majority of particles with a radial position greater than the x-ray spot will not interact with the photons. The radial particle distributions in Figure $4 \mathrm{~b}-\mathrm{d}$ (red lines) show that, additionally to the main peak, smaller outer secondary maxima are present, which reduce the number of particles contained within the interaction volume. The formation of these "wings" has also been observed in other studies of particle distributions from ALS [33, 34, but no explanation as to the source of this effect was given.

To investigate this, we considered the radial distributions of particles within the ALS and show these after the first, third, and fifth lens in Figure $9 \mathrm{a}-\mathrm{c}$, respectively. These were simulated for 2 mbar inlet pressure and 0.31 mbar downstream pressure, with particles evenly distributed at the inlet and neglecting Brownian motion. Example trajectories through the entire ALS are shown in Figure 9 d, It is immediately evident that an outer maximum in the radial distribution is already present after the first lens, and that not all lenses are contracting the particle beam, with some even broadening the distribution. These effects are due to the design of this ALS to accept a large range of particle sizes. In order to visualize the origin of the outer radial maxima we considered particle trajectories through the first lens for different radial starting positions, Figure 10.

A particle exactly on the centerline of the ADL simply stays there, as it feels no radial force, see Figure 8 a. The further off-center the particle is located, the larger the curvature of the flow toward the centerline, leading to a larger radial force. This leads to particles with initial radial positions between $0-2 \mathrm{~mm}$ Figure 10 a) getting pushed towards the center. Due to their inertia particles cross the centerline, but remain closer to it than initially, i. e., the beam is contracted and larger initial radial positions lead to larger final radial positions.

Further away from the centerline the curvature of the flow still increases, while flow speed decreases with proximity to the outer wall (see Figure 8 a). These counter-acting mechanisms negate each other for particles 


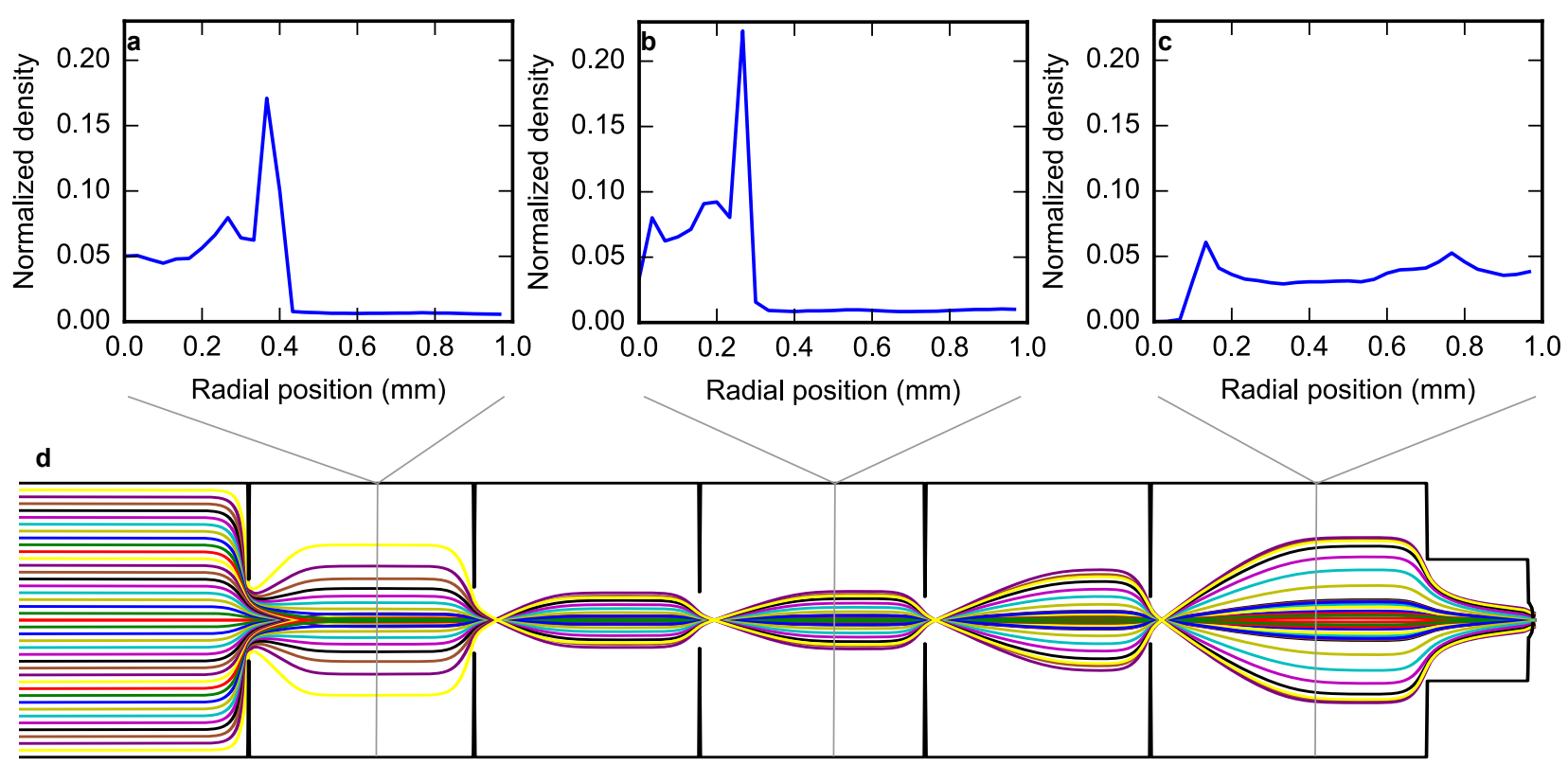

Figure 9: Radial distributions of $500 \mathrm{~nm}$ particles at different stages inside the ALS injector for a uniform initial radial distribution (a-c). The formation of a peak at large radii (i. e., a "wing" in the particle distribution) is already evident after the first lens (a). Example trajectories of $500 \mathrm{~nm}$ particles through the ALS injector, without diffusion effects (d).

with initial radial positions between 2 and $3.5 \mathrm{~mm}($ Figure $10 \mathrm{~b}$ ) and in this region all particles arrive at approximately the same final radial position, regardless of their initial radial position.

Increasing the initial radial position even further $(3.5$ to $5.0 \mathrm{~mm}$, Figure $10 \mathrm{c}$ ) leads to the decreasing flow speed dominating and final radial positions get closer to the centerline with increasing initial radial position, i. e., the opposite effect to that observed in Figure $10 \mathrm{a}$.

Eventually, at around $5.0 \mathrm{~mm}$ (Figure $10 \mathrm{~d}$ ), trajectories stop crossing the centerline and the final radial position increases with increasing initial radial position again. This overall behavior is also summarized in Figure $10 \mathrm{e}$, showing the radial position after the first lens as a function of the initial radial position. The secondary maxima observed in the radial distribution in Figure 4 thus arise at the turning point in $\mathrm{b}$, where several initial radial positions result in the same final position, hence leading to an increased particle density at distinct radii.

This undesirable behavior can be mitigated by designing an ALS such that it only operates in either one of the regimes corresponding to Figure $10 \mathrm{a}$ or d. Total avoidance of secondary maxima can only be accomplished by operating exclusively in regime a, which would - conceptionally - be the best solution to this problem. However, it is experimentally impractical, because it requires an ALS tube much larger than the radial size of the incoming particle beam. Designing a lens such that crossing of the centerline is minimized would ensure that more particles, including those at small initial radii, will be in the regime d, 

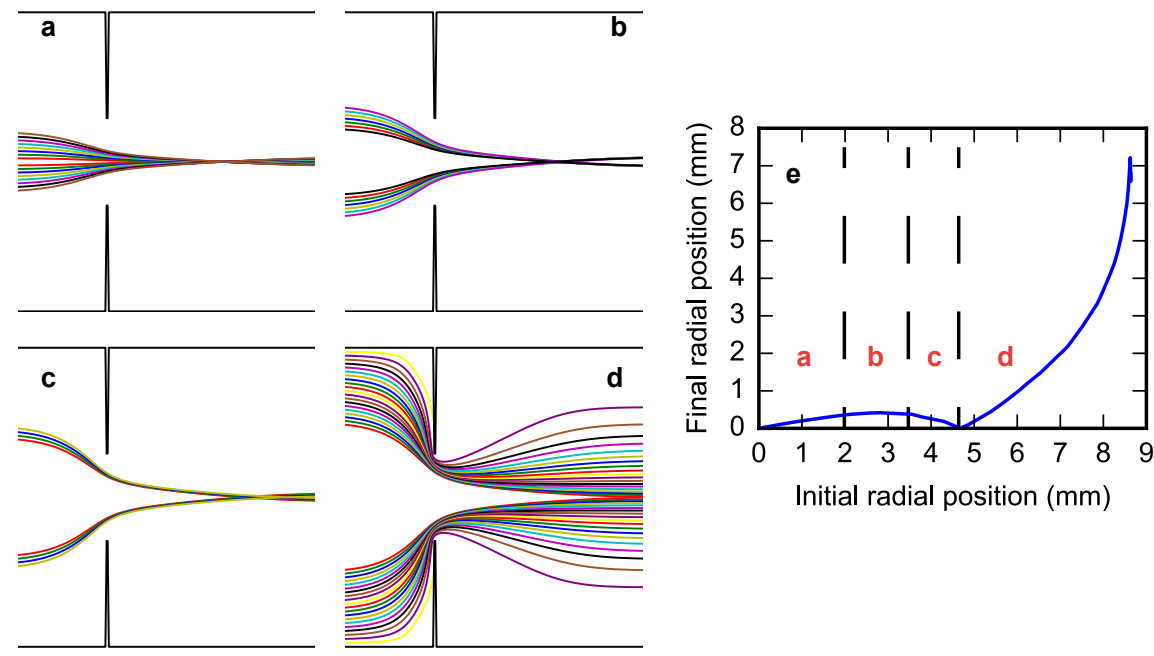

Figure 10: Trajectories of $500 \mathrm{~nm}$ particles through the first lens of the ALS injector, for different radial starting positions of $0-2 \mathrm{~mm}(\mathbf{a}), 2-3.5 \mathrm{~mm}(\mathbf{b}), 3.5-5.0 \mathrm{~mm}(\mathbf{c})$ and $5.0-8.8 \mathrm{~mm}(\mathbf{d})$. Correlation between initial and final radial position for particles traveling through the first lens (e). We identify four distinct regions, as exemplified by the trajectories in $\mathbf{a}-\mathbf{d}$.

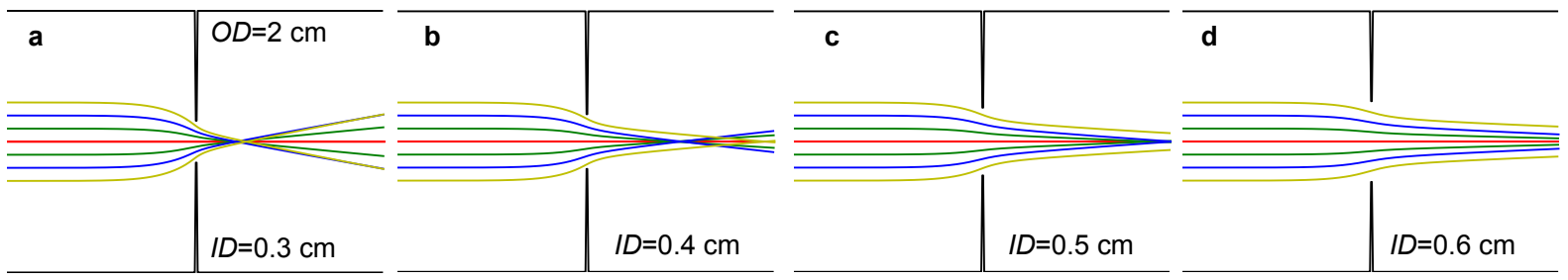

Figure 11: Simulated trajectories of $500 \mathrm{~nm}$ particles through an ADL with an outer diameter of $2 \mathrm{~cm}$ and an inner diameter of $3 \mathrm{~mm}(\mathbf{a}), 4 \mathrm{~mm}(\mathbf{b}), 5 \mathrm{~mm}(\mathbf{c}), 6 \mathrm{~mm}(\mathbf{d})$. The pressure downstream of the ADL is 0.5 mbar and the mass flow of carrier gas is $1.2 \cdot 10^{-2} \mathrm{mg} / \mathrm{min}$.

producing a more collimated particle stream at the output, with fewer particles in secondary maxima, but also with a reduced amount of focusing. This can be achieved by increasing the orifice diameters of the ADL for a given mass flow. This way the absolute value of the derivative of the radial velocity of the gas flow before the orifice with respect to the radial position decreases, while the flow speed drop caused by the walls remains the same. Hence, the radial velocity minimum has an increased value and its position changes to smaller radii, i. e., the minimum in Figure 8 a moves to the upper left. The regimes a-c are moved towards smaller radii until they get negligible. This is highlighted in Figure 11, showing particle trajectories through lenses with various inner diameters for an identical mass flow. Increasing the orifice diameter shifts the crossing point of trajectories further away from the lens and a more collimated particle stream is produced Figure $11 \mathrm{~d}$ ). Thus operation in regime $\mathrm{d}$ is readily achievable, but the corresponding effects of stronger collimation and weaker focusing requires more ADLs for reaching high densities. Balancing these effects for a 

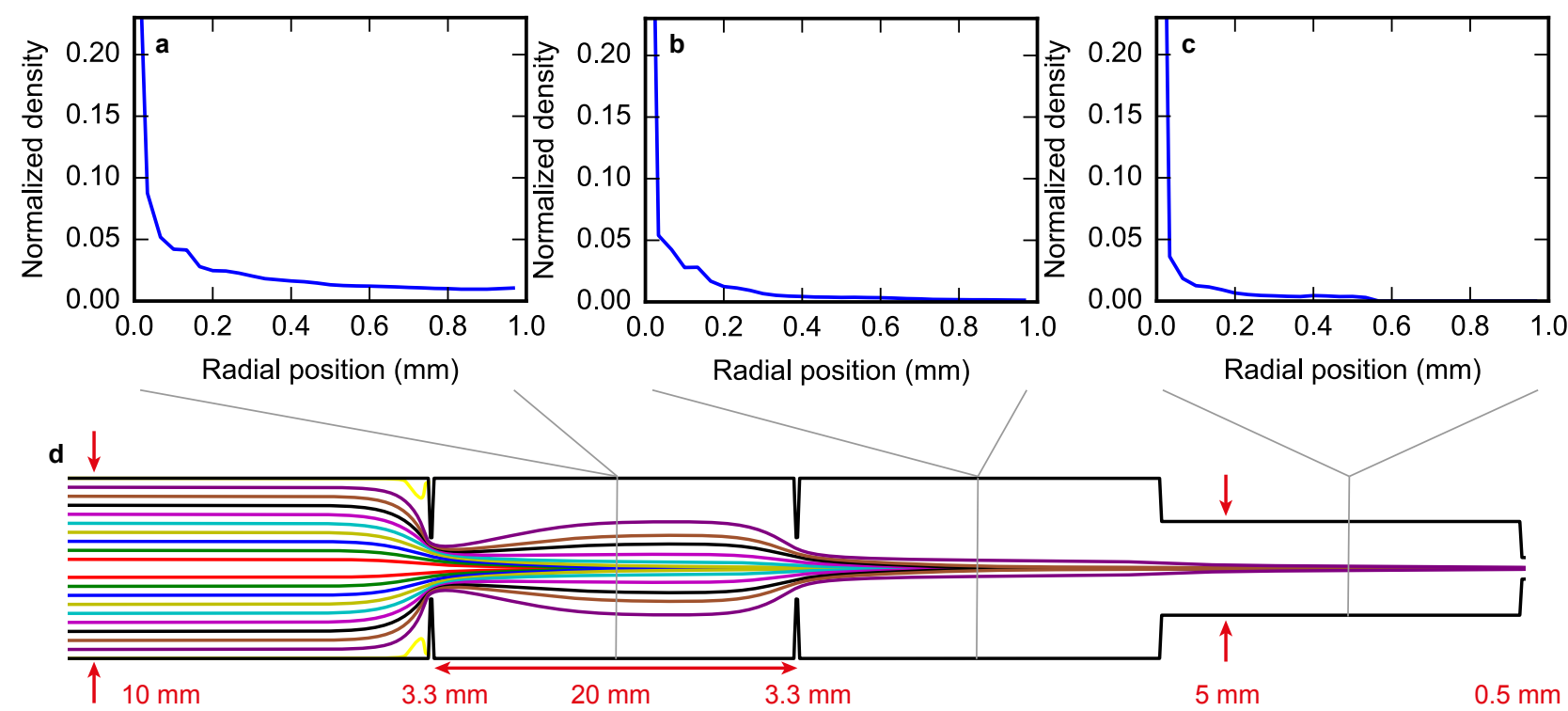

Figure 12: Radial distributions of $500 \mathrm{~nm}$ particles at different stages inside an optimized ALS injector (a-c). The formation of peaks at large radii (i. e., a "wing" in the particle distribution) is significantly reduced in comparison to the conventional ALS in Figure 9 Example trajectories of $500 \mathrm{~nm}$ particles through the optimized ALS injector, without diffusion effects (d).

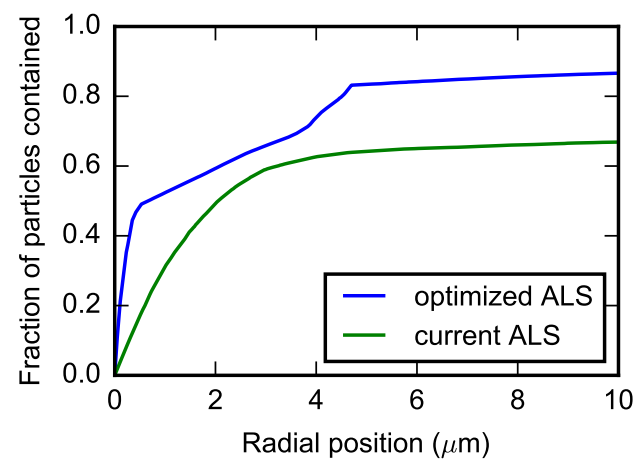

Figure 13: Fraction of particles within a given radius at the position of the respective smallest particle beam waist for the "Uppsala" injector (4.6 mm downstream the exit) and the new ALS design (0.5 mm downstream the exit).

limited amount of ADLs in the ALS, secondary maxima will not completely be avoided when maximizing the central particle density.

Figure 12 shows a three-lens-system for focusing $500 \mathrm{~nm}$ particles that way. Figure $12 \mathrm{a}-\mathrm{c}$ shows radial distributions of $500 \mathrm{~nm}$ particles at various positions within the new ALS, demonstrating that particles are smoothly collimated toward the centerline with significantly weaker secondary maxima than for the conventional ALS in Figure 9, To compare the particle beams of the optimized and the "Uppsala" ALS, we evaluated the fraction of particles arriving within a given radius at the respective particle beam focii, 
$4.6 \mathrm{~mm}$ and $0.5 \mathrm{~mm}$ downstream for the "Uppsala" and optimized injector, respectively; see Figure 13. The optimized ALS exhibits a much steeper increase of the integrated particle fraction at small radii, i. e., focusing a significantly larger fraction of particles into a given radius. As discussed above, secondary maxima cannot be avoided completely in the improved ALS, leading to a kink in the fraction of particles contained around a radius of $\sim 0.5 \mu \mathrm{m}$. In SPI experiments, it is especially pertinent to compare the fraction of particles that would cross the interaction volume with the x-rays. For an x-ray focal spot size with a radius of $0.5 \mu \mathrm{m}$, in the current lens design only $17.1 \%$ of the particles are contained within that radius, while in the optimized design this increases to $48.5 \%$ - corresponding to a nearly threefold improvement. At the same time, the final particle density in the interaction volume also depends on the velocity of the particles leaving the injector. With lower velocities the particles are "packed" closer in the $\mathrm{z}$ dimension and the density is correspondingly higher. For the optimized injector the mean final particle velocity is $43.8 \mathrm{~m} / \mathrm{s}$, whereas for the "Uppsala" injector it is $57.9 \mathrm{~m} / \mathrm{s}$. This leads to a velocity weighted density within the $0.5 \mu \mathrm{m}$ radius spot that is higher by a factor of $\sim 4$ for the optimized injector; for an $\mathrm{x}$-ray focal spot size of $50 \mathrm{~nm}$ radius it would even be higher by a factor of $\sim 9$. While the simulated increase in particle density might not be quantitatively accurate, since this simulation was not taking into account particle diffusion or the skimmer setup, which could significantly influence the final particle beam, see subsection 2.1, it is, nonetheless, clear that an optimized ALS with a compact three-lens design can achieve significantly better particle beam concentration than current injectors.

\section{Conclusion}

The results of a new computer-simulation environment for ALS injectors have been presented. Previous theoretical treatments were quantitatively well reproduced. Focusing on the development of ALS for SPI experiments, the priority is to maximize the particle density along the centerline of the produced particle beam. Comparison of simulated particle profiles with experimental measurements show a good agreement, further validating our computational approach and the ability to describe the experimentally observed behavior. By computing particle trajectories through the ALS, our simulation framework can provide a detailed insight into the particle dynamics inside the ALS, such as the radial position dependent concentration mechanism of an ADL, and hence, the resulting particle profiles. This way we were not only able to monitor, e. g., the overall pressure dependence of the resulting particle beam, but to understand the mechanisms inside an ALS that are responsible for specific artifacts in the radial particle distribution. We could pin down the source of the majority of particle losses in current ALS to be caused by the double skimmer setup before the first lens.

Furthermore, we analyzed the formation of secondary maxima in the radial particle distribution in current ALS and found these to be caused by particles crossing the axial centerline inside the ALS. We 
demonstrated that it is feasible to design a simple ALS that avoids this problem altogether for particles within a narrow size range. It produces a tightly focused stream of particles exhibiting less secondary maxima and a significantly, nearly fourfold, increased particle density at the center of the distribution. In an ALS designed for a wide range of particle sizes the defocusing process and the trapping of particles in secondary maxima cannot be avoided. Therefore, a simple ALS injector system, designed only for a specific particle size, is better suited to fulfill the stringent requirements for atomic-resolution single-particle diffractive imaging and other applications that require highest particle densities. The quick exchange of lenses to adjust for distinct samples would be advantageous for high-throughput experiments. Such an ALS setup is currently under development in our laboratory, along with further quantitative measurements of particle and absolute gas densities emerging from the injector [14, 35], to benchmark and improve simulations by comparison to experiment.

Furthermore, we point out that such an optimized ALS provides a spatial separation of different species that might be present in the original aerosol, similar to more specific separation techniques for small molecules [36, 37], and thus provides a more homogeneous sample for SPI experiments [17].

\section{Acknowledgments}

In addition to DESY, this work has been supported by the European Research Council under the European Union's Seventh Framework Programme (FP7/2007-2013) through the Consolidator Grant COMOTION (ERC-Küpper-614507), by the excellence cluster "The Hamburg Center for Ultrafast Imaging - Structure, Dynamics and Control of Matter at the Atomic Scale" of the Deutsche Forschungsgemeinschaft (CUI, DFG-EXC1074), and by the Helmholtz Gemeinschaft through the "Impuls- und Vernetzungsfond".

\section{References}

[1] M. J. Bogan, W. H. Benner, S. Boutet, U. Rohner, M. Frank, A. Barty, M. M. Seibert, F. Maia, S. Marchesini, S. Bajt, B. Woods, V. Riot, S. P. Hau-Riege, M. Svenda, E. Marklund, E. Spiller, J. Hajdu, H. N. Chapman, Single particle x-ray diffractive imaging, Nano Letters 8 (1) (2008) 310-316. doi:10.1021/n1072728k URL http://pubs.acs.org/cgi-bin/abstract.cgi/nalefd/2008/8/i01/abs/nl072728k.html

[2] M. M. Seibert, T. Ekeberg, F. R. N. C. Maia, M. Svenda, J. Andreasson, O. Jönsson, D. Odić, B. Iwan, A. Rocker, D. Westphal, M. Hantke, D. P. Deponte, A. Barty, J. Schulz, L. Gumprecht, N. Coppola, A. Aquila, M. Liang, T. A. White, A. Martin, C. Caleman, S. Stern, C. Abergel, V. Seltzer, J.-M. Claverie, C. Bostedt, J. D. Bozek, S. Boutet, A. A. Miahnahri, M. Messerschmidt, J. Krzywinski, G. Williams, K. O. Hodgson, M. J. Bogan, C. Y. Hampton, R. G. Sierra, D. Starodub, I. Andersson, S. Bajt, M. Barthelmess, J. C. H. Spence, P. Fromme, U. Weierstall, R. Kirian, M. Hunter, R. B. Doak, S. Marchesini, S. P. Hau-Riege, M. Frank, R. L. Shoeman, L. Lomb, S. W. Epp, R. Hartmann, D. Rolles, A. Rudenko, C. Schmidt, L. Foucar, N. Kimmel, P. Holl, B. Rudek, B. Erk, A. Hömke, C. Reich, D. Pietschner, G. Weidenspointner, L. Strüder, G. Hauser, H. Gorke, J. Ullrich, I. Schlichting, S. Herrmann, G. Schaller, F. Schopper, H. Soltau, K.-U. Kühnel, R. Andritschke, C.-D. Schröter, F. Krasniqi, M. Bott, S. Schorb, D. Rupp, M. Adolph, T. Gorkhover, H. Hirsemann, 
G. Potdevin, H. Graafsma, B. Nilsson, H. N. Chapman, J. Hajdu, Single mimivirus particles intercepted and imaged with an x-ray laser. Nature 470 (7332) (2011) 78. doi:10.1038/nature09748.

URL http://www.nature.com/nature/journal/v470/n7332/full/nature09748.html

[3] R. Neutze, R. Wouts, D. van der Spoel, E. Weckert, J. Hajdu, Potential for biomolecular imaging with femtosecond X-ray pulses, Nature 406 (6797) (2000) 752-757. doi:10.1038/35021099

URL http://dx.doi .org/10.1038/35021099

[4] K. Nass, L. Foucar, T. R. M. Barends, E. Hartmann, S. Botha, R. L. Shoeman, R. B. Doak, R. Alonso-Mori, A. Aquila, S. Bajt, A. Barty, R. Bean, K. R. Beyerlein, M. Bublitz, N. Drachmann, J. Gregersen, H. O. Jönsson, W. Kabsch, S. Kassemeyer, J. E. Koglin, M. Krumrey, D. Mattle, M. Messerschmidt, P. Nissen, L. Reinhard, O. Sitsel, D. Sokaras, G. J. Williams, S. Hau-Riege, N. Timneanu, C. Caleman, H. N. Chapman, S. Boutet, I. Schlichting, Indications of radiation damage in ferredoxin microcrystals using high-intensity X-FEL beams J. Synchrotron Rad. 22 (2) (2015) 225-238. doi: $10.1107 / \mathrm{S} 1600577515002349$. URL http://dx.doi.org/10.1107/S1600577515002349

[5] U. Lorenz, N. M. Kabachnik, E. Weckert, I. A. Vartanyants, Impact of ultrafast electronic damage in single-particle x-ray imaging experiments Phys. Rev. E 86 (2012) 051911. arXiv:1206.6960 doi:10.1103/PhysRevE.86.051911 URL http://link.aps.org/doi/10.1103/PhysRevE.86.051911

[6] B. Ziaja, H. N. Chapman, R. Fäustlin, S. Hau-Riege, Z. Jurek, A. V. Martin, S. Toleikis, F. Wang, E. Weckert, R. Santra, Limitations of coherent diffractive imaging of single objects due to their damage by intense x-ray radiation. New J. Phys. 14 (11) (2012) 115015. doi:10.1088/1367-2630/14/11/115015 URL http://stacks . iop.org/1367-2630/14/i=11/a=115015

[7] M. J. Bogan, S. Boutet, H. N. Chapman, S. Marchesini, A. Barty, W. H. Benner, U. Rohner, M. Frank, S. P. Hau-Riege, S. Bajt, B. Woods, M. M. Seibert, B. Iwan, N. Timneanu, J. Hajdu, J. Schulz, Aerosol imaging with a soft x-ray free electron laser, Aerosol Sci. Techn. 44 (3) (2010) i-vi. doi:10.1080/02786820903485800 URL http://dx.doi.org/10.1080/02786820903485800

[8] T. Ekeberg, M. Svenda, C. Abergel, F. R. N. C. Maia, V. Seltzer, J.-M. Claverie, M. Hantke, O. Jönsson, C. Nettelblad, G. van der Schot, M. Liang, D. P. Deponte, A. Barty, M. M. Seibert, B. Iwan, I. Andersson, N. D. Loh, A. V. Martin, H. Chapman, C. Bostedt, J. D. Bozek, K. R. Ferguson, J. Krzywinski, S. W. Epp, D. Rolles, A. Rudenko, R. Hartmann, N. Kimmel, J. Hajdu, Three-dimensional reconstruction of the giant mimivirus particle with an x-ray free-electron laser. Phys. Rev. Lett. 114 (9) (2015) 098102. doi:https://doi.org/10.1103/PhysRevLett.114.098102 URL http://dx.doi.org/10.1103/PhysRevLett.114.098102

[9] H. N. Chapman, X-ray imaging beyond the limits Nature Mater. 8 (4) (2009) 299-301. doi:10.1038/nmat2402 URL http://dx.doi.org/10.1038/nmat2402

[10] D. P. DePonte, U. Weierstall, K. Schmidt, J. Warner, D. Starodub, J. C. H. Spence, R. B. Doak, Gas dynamic virtual nozzle for generation of microscopic droplet streams J. Phys. D 41 (19) (2008) 195505. doi:10.1088/0022-3727/41/19/195505 URL http://iopscience .iop.org/0022-3727/41/19/195505

[11] S. Awel, R. A. Kirian, M. O. Wiedorn, K. R. Beyerlein, N. Roth, D. A. Horke, D. Oberthür, J. Knoska, V. Mariani, A. Morgan, L. Adriano, A. Tolstikova, P. L. Xavier, O. Yefanov, A. Aquila, A. Barty, S. Roy-Chowdhury, M. S. Hunter, D. James, J. S. Robinson, U. Weierstall, A. V. Rode, S. Bajt, J. Küpper, H. N. Chapman, Femtosecond X-ray diffraction from an aerosolized beam of protein nanocrystals J. Appl. Cryst. 51 (1) (2018) 133-139. arXiv:1702.04014 doi:10.1107/S1600576717018131 URL https://doi.org/10.1107/S1600576717018131

[12] M. F. Hantke, D. Hasse, M. R. N. C, T. Ekeberg, K. John, M. Svenda, N. D. Loh, A. V. Martin, N. Timneanu, L. S. D, van der SchotGijs, G. H. Carlsson, M. Ingelman, J. Andreasson, D. Westphal, M. Liang, F. Stellato, D. P. DePonte, R. Hartmann, N. Kimmel, R. A. Kirian, M. M. Seibert, K. Mühlig, S. Schorb, K. Ferguson, C. Bostedt, S. Carron, J. D. 
Bozek, D. Rolles, A. Rudenko, S. Epp, H. N. Chapman, A. Barty, J. Hajdu, I. Andersson, High-throughput imaging of heterogeneous cell organelles with an x-ray laser. Nat. Photon. 8 (12) (2014) 943-949. doi:doi:10.1038/nphoton.2014.270 URL http://www.nature.com/nphoton/journal/v8/n12/full/nphoton.2014.270.html

[13] R. A. Kirian, S. Awel, N. Eckerskorn, H. Fleckenstein, M. Wiedorn, L. Adriano, S. Bajt, M. Barthelmess, R. Bean, K. R. Beyerlein, L. M. G. Chavas, M. Domaracky, M. Heymann, D. A. Horke, J. Knoska, M. Metz, A. Morgan, D. Oberthuer, N. Roth, T. Sato, P. L. Xavier, O. Yefanov, A. V. Rode, J. Küpper, H. N. Chapman, Simple convergentnozzle aerosol injector for single-particle diffractive imaging with x-ray free-electron lasers Struct. Dyn. 2 (4) (2015) 041717. doi:10.1063/1.4922648

URL http://dx.doi.org/10.1063/1.4922648

[14] S. Awel, R. A. Kirian, N. Eckerskorn, M. Wiedorn, D. A. Horke, A. V. Rode, J. Küpper, H. N. Chapman, Visualizing aerosolparticle injection for diffractive-imaging experiments, Opt. Exp. 24 (6) (2016) 6507-6521. doi:10.1364/0E.24.006507 URL http://dx.doi.org/10.1364/0E.24.006507

[15] M. Bergh, G. Huldt, N. Tîmneanu, F. R. N. C. Maia, J. Hajdu, Feasibility of imaging living cells at subnanometer resolutions by ultrafast x-ray diffraction, Quarterly Reviews of Biophysics 41 (2008) 181-204. doi:10.1017/S003358350800471X URL http://journals.cambridge.org/article_S003358350800471X

[16] R. Fung, A. M. Hanna, O. Vendrell, S. Ramakrishna, T. Seideman, R. Santra, A. Ourmazd, Dynamics from noisy data with extreme timing uncertainty, Nature 532 (7600) (2016) 471-475. doi:10.1038/nature17627 URL http://dx.doi.org/10.1038/nature17627

[17] A. Barty, J. Küpper, H. N. Chapman, Molecular imaging using x-ray free-electron lasers, Annu. Rev. Phys. Chem. 64 (1) (2013) 415-435. doi:10.1146/annurev-physchem-032511-143708 URL http://dx.doi .org/10.1146/annurev-physchem-032511-143708

[18] A. Robinson, On the motion of small particles in a potential field of flow, Comm. Pure Appl. Math. 9 (1) (1956) 69-84. doi:10.1002/cpa.3160090105. URL http://onlinelibrary.wiley.com/doi/10.1002/cpa.3160090105/abstract

[19] P. Liu, P. J. Ziemann, D. B. Kittelson, P. H. McMurry, Generating particle beams of controlled dimensions and divergence: I. theory of particle motion in aerodynamic lenses and nozzle expansions Aerosol Sci. Techn. 22 (3) (1995) $293-313$. doi: $10.1080 / 02786829408959748$. URL http://dx.doi.org/10.1080/02786829408959748

[20] X. F. Zhang, K. A. Smith, D. R. Worsnop, J. Jimenez, J. T. Jayne, C. E. Kolb, A numerical characterization of particle beam collimation by an aerodynamic lens-nozzle system: Part I. An individual lens or nozzle, Aerosol Sci. Techn. 36 (5) (2002) 617-631. doi:10.1080/02786820252883856 URL http://www.tandfonline.com/doi/abs/10.1080/02786820252883856

[21] X. Zhang, K. A. Smith, D. R. Worsnop, J. L. Jimenez, J. T. Jayne, C. E. Kolb, J. Morris, P. Davidovits, Numerical Characterization of Particle Beam Collimation: Part II Integrated Aerodynamic-Lens-Nozzle System, Aerosol Sci. Techn. 38 (6) (2004) 619-638. doi:10.1080/02786820490479833 URL http://www.tandfonline.com/doi/abs/10.1080/02786820490479833

[22] X. Wang, A. Gidwani, S. L. Girshick, P. H. McMurry, Aerodynamic Focusing of Nanoparticles: II. Numerical Simulation of Particle Motion Through Aerodynamic Lenses Aerosol Sci. Techn. 39 (7) (2005) 624-636. doi:10.1080/02786820500181950 URL http://www .tandfonline.com/doi/abs/10.1080/02786820500181950

[23] X. Wang, P. H. McMurry, A design tool for aerodynamic lens systems, Aerosol Sci. Technol. 40 (5) (2006) $320-334$. doi:10.1080/02786820600615063. URL http://dx.doi.org/10.1080/02786820600615063

[24] K.-S. Lee, S.-W. Cho, D. Lee, Development and experimental evaluation of aerodynamic lens as an aerosol inlet of single 
particle mass spectrometry, J. Aerosol Sci. 39 (4) (2008) 287-304. doi:10.1016/j.jaerosci.2007.10.011

URL http://dx.doi.org/10.1016/j·jaerosci.2007.10.011

[25] J. Meinen, S. Khasminskaya, E. Rühl, W. Baumann, T. Leisner, The TRAPS apparatus: Enhancing target density of nanoparticle beams in vacuum for x-ray and optical spectroscopy, Aerosol Sci. Techn. 44 (4) (2010) 316-328. doi: $10.1080 / 02786821003639692$

URL http://www.tandfonline.com/doi/abs/10.1080/02786821003639692

[26] S. Zherebtsov, T. Fennel, J. Plenge, E. Antonsson, I. Znakovskaya, A. Wirth, O. Herrwerth, F. Süßmann, C. Peltz, I. Ahmad, S. A. Trushin, V. Pervak, S. Karsch, M. J. J. Vrakking, B. Langer, C. Graf, M. I. Stockman, F. Krausz, E. Rühl, M. F. Kling, Controlled near-field enhanced electron acceleration from dielectric nanospheres with intense few-cycle laser fields, Nat. Phys. 7 (8) (2011) 656-662. doi:10.1038/nphys1983

URL http://dx.doi.org/10.1038/nphys1983

[27] M. R. Canagaratna, J. T. Jayne, J. L. Jimenez, J. D. Allan, M. R. Alfarra, Q. Zhang, T. B. Onasch, F. Drewnick, H. Coe, A. Middlebrook, A. Delia, L. R. Williams, A. M. Trimborn, M. J. Northway, P. F. DeCarlo, C. E. Kolb, P. Davidovits, D. R. Worsnop, Chemical and microphysical characterization of ambient aerosols with the aerodyne aerosol mass spectrometer. Mass Spectrom. Rev. 26 (2) (2007) 185-222. doi:10.1002/mas.20115

URL http://dx.doi.org/10.1002/mas.20115

[28] COMSOL Multiphysics v. 5.3. http://www.comsol.com COMSOL AB, Stockholm, Sweden.

[29] D. K. Hutchins, M. H. Harper, R. L. Felder, Slip correction measurements for solid spherical particles by modulated dynamic light scattering, Aerosol Sci. Techn. 22 (2) (1995) 202-218. doi:10.1080/02786829408959741 URL http://www.tandfonline.com/doi/abs/10.1080/02786829408959741

[30] A. Li, G. Ahmadi, Dispersion and deposition of spherical particles from point sources in a turbulent channel flow, Aerosol Sci. Techn. 16 (24) (1992) 209-226. doi:10.1080/02786829208959550 URL http://www.tandfonline.com/doi/abs/10.1080/02786829208959550

[31] K. R. Beyerlein, L. Adriano, M. Heymann, R. Kirian, J. Knoska, F. Wilde, H. N. Chapman, S. Bajt, Ceramic microinjection molded nozzles for serial femtosecond crystallography sample delivery Rev. Sci. Instrum. 86 (12) (2015) 125104-12. doi:10.1063/1.4936843

URL http://dx.doi.org/10.1063/1.4936843

[32] J. Bielecki, private communication (2017).

[33] G. A. Petrucci, P. B. Farnsworth, P. Cavalli, N. Omenetto, A differentially pumped particle inlet for sampling of atmospheric aerosols into a time-of-flight mass spectrometer: Optical characterization of the particle beam Aerosol Sci. Techn. 33 (1-2) (2000) 105-121. doi:10.1080/027868200410877 URL http://dx.doi.org/10.1080/027868200410877

[34] J. M. Headrick, P. E. Schrader, H. A. Michelsen, Radial-profile and divergence measurements of combustion-generated soot focused by an aerodynamic-lens system J. Aerosol Sci. 58 (2013) 158-170. doi:http://dx.doi.org/10.1016/j.jaerosci. 2013.01 .002

URL http://www.sciencedirect.com/science/article/pii/S0021850213000062

[35] D. A. Horke, N. Roth, L. Worbs, J. Küpper, Characterizing gas flow from aerosol particle injectors, J. Appl. Phys. 121 (12) (2017) 123106. arXiv:1609.09020 doi:10.1063/1.4978914 URL http://dx.doi.org/10.1063/1.4978914

[36] F. Filsinger, U. Erlekam, G. von Helden, J. Küpper, G. Meijer, Selector for structural isomers of neutral molecules, Phys. Rev. Lett. 100 (2008) 133003. arXiv:0802.2795, doi:10.1103/PhysRevLett.100.133003 URL http://dx.doi.org/10.1103/PhysRevLett.100.133003

[37] Y.-P. Chang, D. A. Horke, S. Trippel, J. Küpper, Spatially-controlled complex molecules and their applications, Int. Rev. 
Phys. Chem. 34 (2015) 557-590. arXiv:1505.05632 doi:10.1080/0144235X.2015.1077838

URL http://dx.doi.org/10.1080/0144235X.2015.1077838 